\title{
Abundance structure and chemical evolution of the Galactic disc
}

\author{
Thomas Bensby $^{1}$ and Sofia Feltzing ${ }^{2}$ \\ ${ }^{1}$ European Southern Observatory, Alonso de Cordova 3107, Vitacura, Santiago, Chile \\ email: tbensby@eso.org \\ ${ }^{2}$ Lund Observatory, Box 43, SE-22100 Lund, Sweden \\ email: sofia@astro.lu.se
}

\begin{abstract}
We have obtained high-resolution, high signal-to-noise spectra for $899 \mathrm{~F}$ and $\mathrm{G}$ dwarf stars in the Solar neighbourhood. The stars were selected on the basis of their kinematic properties to trace the thin and thick discs, the Hercules stream, and the metal-rich stellar halo. A significant number of stars with kinematic properties 'in between' the thin and thick discs were also observed to investigate in greater detail the dichotomy of the Galactic disc. All stars have been homogeneously analysed, using the exact same methods, atomic data, model atmospheres, etc., and also truly differentially to the Sun. Hence, the sample is likely to be free from internal errors, allowing us to, in a multi-dimensional space consisting of detailed elemental abundances, stellar ages, and the full three-dimensional space velocities, reveal very small differences between the stellar populations.
\end{abstract}

Keywords. stars: abundances, stars: kinematics, Galaxy: disk, Galaxy: evolution,

Compared to our previous studies of the Galactic thin and thick discs (Bensby et al. $2003,2005)$ the current stellar sample is larger by a factor of $\sim 8$. The figure shows the thin and thick disc abundance trends based on kinematical selection criteria only. The red full line in each plot is the running median from the thick disc stars, and the dashed blue line the running median from the thin disc stars. It is clear that there is separation between the two discs up to at least solar metallicities, signaling the dichotomy of the Galactic stellar disc, and that the two discs have had very different chemical histories. First results, based on this enlarged sample, regarding the origin of the Hercules stream and the metal-rich limit of the thick disc were published in Bensby et al. (2007a,b). The full data set will be published in the fourth quarter of 2009 where we will investigate in great detail the abundance structure and chemical evolution of the Galactic stellar disc.
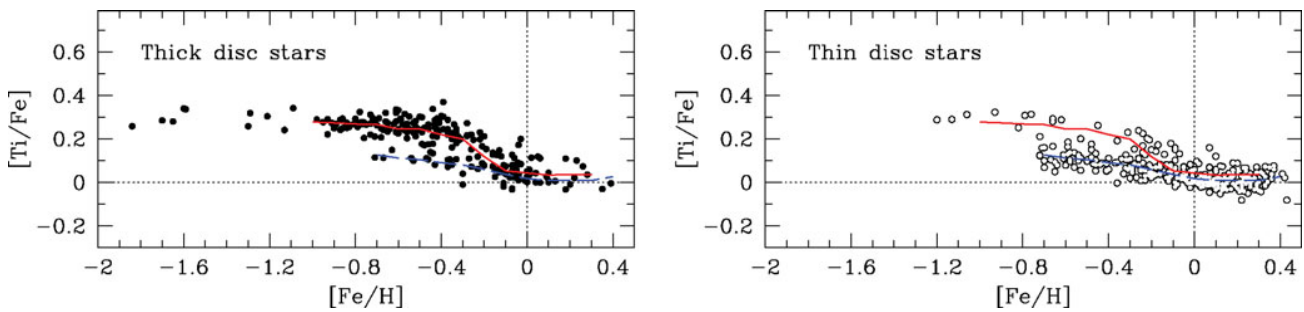

\section{References}

Bensby, T., Feltzing, S., \& Lundström, I. 2003, $A \mathscr{E} A$, 410, 527

Bensby, T., Feltzing, S., Lundström, I., \& Ilyin, I. 2005, A $\mathscr{E} A, 415,155$

Bensby, T., Oey, M. S., Feltzing, S., \& Gustafsson, B. 2007a, ApJ, 655, L89

Bensby, T., Zenn, A. R., Oey, M. S., \& Feltzing, S. 2007b, ApJ, 663, L13 